\title{
SOME RESULTS ON CASCADE DISCRETE-TIME SYSTEMS
}

XIAO-MING BAI, HUI-MIN LI, AND XIAO-SONG YANG

Received 16 September 2005; Accepted 5 December 2005

We present sufficient conditions for global asymptotic stability of cascade discrete-time systems. Considering failure of the global asymptotic stability in some cascade systems, we give an estimate of the region of attraction of the systems.

Copyright (c) 2006 Xiao-Ming Bai et al. This is an open access article distributed under the Creative Commons Attribution License, which permits unrestricted use, distribution, and reproduction in any medium, provided the original work is properly cited.

\section{Introduction}

Cascade systems have received much attention (see [1-6]). A currently active area of research in cascade systems is the study of the discrete-time systems, because a lot of cascade continuous-time systems are usually discretized in engineering as sampled-data systems. Hence in this paper we focus on the cascade discrete-time systems given by

$$
\begin{gathered}
x(n+1)=f(x(n), y(n)), \\
y(n+1)=g(y(n)), \quad x \in \mathbb{R}^{n}, y \in \mathbb{R}^{m},
\end{gathered}
$$

where $f$ and $g$ are assumed to be $C^{1}$ mapping with $f(0,0)=0$ and $g(0)=0$.

To conveniently study some properties of the system (1.1) in detail, we rewrite the above system in the following form:

$$
\begin{aligned}
& x(n+1)=\bar{f}(x(n))+h(x(n), y(n)), \\
& y(n+1)=g(y(n)), \quad x \in \mathbb{R}^{n}, y \in \mathbb{R}^{m},
\end{aligned}
$$

where $\bar{f}$ and $h$ satisfy the following conditions:

$$
\begin{gathered}
\bar{f}(x(n))=f(x(n), 0), \\
h(x(n), y(n))=f(x(n), y(n))-f(x(n), 0), \quad \bar{f}(0)=0, h(x, 0)=0 .
\end{gathered}
$$


Throughout this paper we make the following standing assumptions for (1.1) or (1.2). Assumption 1.1. The equilibrium point $x=0$ of (1.4) is globally asymptotically stable:

$$
x(n+1)=f(x(n), 0)=\bar{f}(x(n)) .
$$

Assumption 1.2. The equilibrium point $y=0$ of (1.5) is globally asymptotically stable:

$$
y(n+1)=g(y(n))
$$

A main problem is the stability of the equilibrium point $o=(0,0)$ with respect to the system (1.1) or (1.2). A well-known result on this problem was obtained in [2].

Theorem 1.3. Suppose that Assumptions 1.1 and 1.2 hold, and every solution to (1.1) or (1.2) is bounded, then the equilibrium point $o=(0,0)$ of (1.1) or (1.2) is globally asymptotically stable.

The result may be the most general statement of cascade systems without taking into consideration the $x$-subsystem in (1.1) or (1.2). However, the boundedness of solutions to (1.1) or (1.2) is very hard to verify. Therefore a key problem is what conditions may be used to replace the boundedness of solutions. To get an insight into this problem, Yang [6] studied what structure and growth rate of the connection term can fail the boundedness of solutions of a cascade system. In addition, it was proposed in [6] that the conditions $\|h(x, y)\| \leq k(y)(\bar{h}+h\|x\|)$, where $\bar{h}$ and $h$ are two positive constants and $k(y)$ is a continuous function with $k(0)=0$, together with an additional condition should be sufficient to guarantee the boundedness of solutions to (1.2) under Assumptions 1.1 and 1.2. However the concrete additional condition was not presented in [6]. In this paper we will give such an additional condition.

The paper is organized as follows. In Section 2 we give Theorem 2.1 as an answer to the above question. In Section 3 an approximate theorem is obtained for a specific class of time-varying systems, in which we discuss that every solution of the system can be approximated by the corresponding zero-input system and estimate convergence rate. Finally we present an interesting result that gives an estimate of the region of attraction of the system (1.2) under Assumptions 1.1 and 1.2.

\section{Further result on global asymptotic stability}

In this section we study the problem mentioned in the introduction, and give the following theorem whose key point is to put some conditions on $\bar{f}$.

Theorem 2.1. Suppose that Assumptions 1.1 and 1.2 hold, and

(a) $\varlimsup_{\|x\| \rightarrow \infty}(\|\bar{f}(x)\| /\|x\|)=\mu<1$,

(b) $\|h(x, y)\| \leq k(y)(\bar{h}+h\|x\|)$, where $h$ and $\bar{h}$ are two positive constants and $k(y)$ is a continuous function with $k(0)=0$,

then the equilibrium point of the system (1.2) is globally asymptotically stable. 
Proof. According to Theorem 1.3, if every solution of the system (1.2) is bounded, Theorem 2.1 holds. So we only prove the boundedness of solutions to (1.2). For this purpose, we suppose that the trajectory $x(n), n \in \mathbb{N}$, of $x$-subsystem with certain given initial value $x_{0}, y_{0}$ is unbounded.

Since $\overline{\lim }_{\|x\| \rightarrow \infty}(\|\bar{f}(x)\| /\|x\|)=\mu<1$, for $\varepsilon_{1}=(1+\mu) / 2$, there exists a positive constant $\eta$ that satisfies $\eta>\bar{h}$ such that if $\|x\| \geq \eta$, then

$$
\|\bar{f}(x)\|<\varepsilon_{1}\|x\| .
$$

Since $\|\bar{f}(x)\|$ is continuous, we define a continuous function $G: \mathbb{R}^{n} \rightarrow \mathbb{R}$ by

$$
G(x)=\|\bar{f}(x)\|
$$

It is easy to see that $\max _{\|x\| \leq \eta} G(x)=b<+\infty$. Let $A=G^{-1}(b)$ and $\lambda=\max _{x \in A}\|x\|$.

By Assumption 1.2, for the given initial value $y_{0}$, we have

$$
\lim _{n \rightarrow \infty} k(y(n))=k(0)=0 .
$$

It follows that for $\varepsilon_{2}=\left(1-\varepsilon_{1}\right) / 2(h+1)$, there exists $\bar{N}\left(y_{0}\right)>0$ such that

$$
k(y(n))<\varepsilon_{2}, \quad \forall n>\bar{N}\left(y_{0}\right) .
$$

Now we establish the following obvious inequality, which is useful in proving the statement. If $\|x(n)\|>\eta$ and $k(y(n))<\varepsilon_{2}$, then

$$
\begin{aligned}
\|x(n+1)\| & \leq\|\bar{f}(x(n))\|+k(y)(\bar{h}+h\|x(n)\|) \\
& \leq \varepsilon_{1}\|x(n)\|+\frac{1-\varepsilon_{1}}{2}\|x(n)\|=\frac{\mu+3}{4}\|x(n)\| .
\end{aligned}
$$

Since the trajectory $x(n)$ of $x$-subsystem with initial value $x_{0}, y_{0}$ is unbounded, there exists a positive constant $\tilde{N}\left(x_{0}\right)$ that satisfies $\tilde{N}\left(x_{0}\right)>\bar{N}\left(y_{0}\right)$ such that

$$
\left\|x\left(\tilde{N}\left(x_{0}\right)\right)\right\|>\eta
$$

In view of (2.5) we have that if $n>\tilde{N}\left(x_{0}\right)$, then

$$
\|x(n)\| \leq \max \left(\left\|x\left(\tilde{N}\left(x_{0}\right)\right)\right\|, b+\frac{1-\varepsilon_{1}}{2}(\bar{h}+h \lambda)\right)
$$

showing that the trajectory $x(n)$ is bounded, which is a contradiction to the above hypothesis.

Therefore all solutions to (1.2) are bounded. The proof is complete.

Remark 2.2. The following example shows that condition (a) in Theorem 2.1 is "necessary" when condition (b) holds under Assumptions 1.1 and 1.2. 
4 Some results on cascade discrete-time systems

Example 2.3.

$$
\begin{gathered}
x(n+1)=\bar{f}(x(n))+4 x(n) y(n), \\
y(n+1)=g(y(n)), \quad x \in \mathbb{R}, y \in \mathbb{R},
\end{gathered}
$$

where

$$
\bar{f}(x)=\left\{\begin{array}{ll}
\frac{x^{2}}{1-x}, & x<0, \\
\frac{x^{2}}{1+x}, & x \geq 0,
\end{array} \quad g(y)= \begin{cases}\frac{y}{1-y}, & y<0, \\
\frac{y}{1+y}, & y \geq 0,\end{cases}\right.
$$

and $h(x, y)=4 x y$ with $k(y)=|y|, \bar{h}=0$ and $h=4$.

In the above system, it is easy to verify that the other conditions in Theorem 2.1 hold except for condition (a) and to prove that there exist $x_{0}=1, y_{0}=1$ such that when $n \geq 1$, $x(n)>n+1$, which implies that the trajectory with $x_{0}=1, y_{0}=1$ is unbounded.

\section{An approximate theorem}

This section is devoted to studying the convergence rate of solutions of the nonlinear system that can be described by

$$
x(n+1)=f(x(n), y(n)), \quad x \in \mathbb{R}^{n}, y \in \mathbb{R}^{m},
$$

where $f(0,0)=0$ and $y$ is an input that converges to zero. Usually the system (3.1) is complicated, so we hope to estimate the convergence rate of solutions of (3.1) by studying the convergence rate of solutions of its zero-input system

$$
x(n+1)=f(x(n), 0)=\bar{f}(x(n)), \quad x \in \mathbb{R}^{n}
$$

with $\bar{f}(0)=0$.

When the input $y(n)$ satisfies an appropriate exponential bound, we consider the particular case of (3.1) when the input enters additively, that is,

$$
x(n+1)=\bar{f}(x(n))+c y(n), \quad x \in \mathbb{R}^{n}, y \in \mathbb{R}^{m},
$$

where $c \in \mathbb{R}^{n \times m}$ is a constant matrix.

Before discussing the system (3.3), we assume that the following condition is satisfied: $C_{1}: \bar{f}: \mathbb{R}^{n} \rightarrow \mathbb{R}^{n}$ is a contraction mapping, that is,

$$
\left\|\bar{f}\left(x_{1}\right)-\bar{f}\left(x_{2}\right)\right\| \leq r\left\|x_{1}-x_{2}\right\|, \quad 0<r<1, \forall x_{1}, x_{2} \in \mathbb{R}^{n} .
$$

In the following arguments, let $\bar{x}(n)$ denote the trajectory of (3.2) and let $x(n)$ denote the trajectory of (3.3).

The following approximation theorem shows that if $y(n)$ satisfies an exponential bound, that is,

$$
\|y(n)\| \leq \kappa a^{n}, \quad \text { for } n \geq 0,
$$


where $\kappa$ is a positive constant and $0<a<1$, then every trajectory $x(n)$ is approximated by the trajectory corresponding to the zero input, in the sense that

$$
\|x(n)-\bar{x}(n)\|<\varepsilon \eta^{n}, \quad \forall \varepsilon>0, \eta=\frac{\max (r, a)+1}{2},
$$

provided that $n$ is sufficiently large.

Theorem 3.1. Suppose that $C_{1}$ holds, and $y(n)$ is an $\mathbb{R}^{m}$-valued function such that the bound (3.5) holds, then for every trajectory of (3.3) there is a trajectory of (3.2) such that (3.6) is satisfied.

Proof. The difference between the trajectories $\bar{x}(n)$ and $x(n)$ with the same initial value $x_{0}$ satisfies

$$
\begin{aligned}
\|x(n+1)-\bar{x}(n+1)\| & =\|\bar{f}(x(n))+c y(n)-\bar{f}(\bar{x}(n))\| \\
& \leq\|\bar{f}(x(n))-\bar{f}(\bar{x}(n))\|+\|c\|\|y(n)\|,
\end{aligned}
$$

where $\|c\|=\max _{1 \leq i \leq n} \max _{1 \leq j \leq m}\left|c_{i j}\right|$.

Using condition $C_{1}$, we have

$$
\|x(n+1)-\bar{x}(n+1)\| \leq r\|x(n)-\bar{x}(n)\|+\|c\|\|y(n)\| .
$$

Letting $b(n)=\|x(n)-\bar{x}(n)\|$, one has

$$
b(n+1) \leq r b(n)+\|c\|\|y(n)\| \leq r b(n)+\|c\| \kappa a^{n} .
$$

Subsequently, we have

$$
\frac{b(n+1)}{\eta^{n+1}} \leq \frac{r}{\eta} \frac{b(n)}{\eta^{n}}+\|c\| \kappa \frac{a^{n}}{\eta^{n+1}} .
$$

Letting $z(n)=b(n) / \eta^{n}$, we get

$$
z(n+1) \leq \frac{r}{\eta} z(n)+\|c\| \kappa \frac{a^{n}}{\eta^{n+1}} .
$$

Furthermore, we easily have

$$
S(n+1) \leq \frac{r}{\eta} S(n)+\sum_{i=0}^{n}\|c\| \kappa \frac{a^{n}}{\eta^{n+1}} \leq \frac{r}{\eta} S(n)+\sigma,
$$

where $S(n)=\sum_{i=0}^{n} z(n)$ and $\sigma=\sum_{i=0}^{\infty}\|c\| \kappa\left(a^{n} / \eta^{n+1}\right)$.

Since $S(n) \leq S(n+1)$, one has

$$
\begin{gathered}
S(n) \leq S(n+1) \leq \frac{r}{\eta} S(n)+\sigma, \\
S(n) \leq \frac{\sigma \eta}{\eta-r} .
\end{gathered}
$$


6 Some results on cascade discrete-time systems

By monotone convergence theorem, we have that $S(n)$ is convergent, which implies that

$$
\lim _{n \rightarrow \infty} z(n)=0 .
$$

Therefore (3.6) holds. The proof is complete.

Now we study a generalization of the above result and consider the cascade system $(1.2)$

$$
\begin{aligned}
& x(n+1)=\bar{f}(x(n))+h(x(n), y(n)) \\
& y(n+1)=g(y(n)), \quad x \in \mathbb{R}^{n}, y \in \mathbb{R}^{m},
\end{aligned}
$$

where $h(x, y)$ also satisfies the following condition:

$$
\left\|h\left(x(n), y_{1}(n)\right)-h\left(x(n), y_{2}(n)\right)\right\| \leq m\left\|y_{1}(n)-y_{2}(n)\right\|^{\theta}, \quad \forall y_{1}, y_{2} \in \mathbb{R}^{m},
$$

where $\theta$ and $m$ are two positive constants.

Before stating our result, we also make the following assumption:

$$
C_{2}: g: \mathbb{R}^{m} \longrightarrow \mathbb{R}^{m} \quad \text { satisfies }\left\|g\left(y_{1}\right)-g\left(y_{2}\right)\right\| \leq r^{\prime}\left\|y_{1}-y_{2}\right\|, 0<r^{\prime}<1, \forall y_{1}, y_{2} \in \mathbb{R}^{m}
$$

and (3.6) should be written as follows:

$$
\|x(n)-\bar{x}(n)\| \leq \varepsilon \bar{\eta}^{n}, \quad \forall \varepsilon>0, \bar{\eta}=\frac{\max \left(r, r^{\prime}\right)+1}{2},
$$

provided that $n$ is sufficiently large.

Now, by the above similar arguments we give the following result.

Theorem 3.2. Suppose that $C_{1}$ and $C_{2}$ hold, then for every solution of (3.3) there is a trajectory corresponding to the zero input such that (3.18) is satisfied.

Remark 3.3. If the input is globally exponentially stable, we will get a similar result.

\section{An estimate of the region of attraction}

Consider the cascade system (1.2), that is,

$$
\begin{gathered}
x(n+1)=\bar{f}(x(n))+h(x(n), y(n))=f(x(n), y(n)), \\
y(n+1)=g(y(n)), \quad x \in \mathbb{R}^{n}, y \in \mathbb{R}^{m},
\end{gathered}
$$

under Assumptions 1.1 and 1.2.

From [2], we know that under the above assumptions the system (1.2) is likely not globally attractive, but the local region of attraction of the system (1.2) is existent. On the basis of the above result we have the following theorem. 
Theorem 4.1. Suppose that Assumptions 1.1, 1.2, and $C_{1}$ hold. Then for any bounded open set $U=\{x \mid\|x\|<\rho\}$, where $\rho$ is a positive constant, there exists a neighborhood $V$ of $y=$ $0 \in \mathbb{R}^{m}$ such that $U \times V=\{(x, y) \mid x \in U, y \in V\}$ is the region of attraction of (1.2).

Proof. Since condition $C_{1}$ holds and $\bar{f}$ is a $C^{1}$ function, it follows that

$$
\|D \bar{f}\|=\|D f(x, 0)\| \leq r<1 .
$$
by

Denoting $U=\{x \mid\|x\|<\rho\}$, we define $I: \mathbb{R}^{m} \rightarrow \mathbb{R}$ to be a continuous function defined

$$
I(y)=\max _{x \in \bar{U}}\left\|\partial_{x} f(x, y)\right\|
$$

where $\bar{U}$ is the closure of the set $U$.

Thus the continuity of $I(y)$ ensures the existence of a neighborhood $V^{\prime}$ of $y=0$ such that if $y \in V^{\prime}$, then $I(y)<(r+1) / 2$.

Since $f$ is a $C^{1}$ mapping, we let $d=\max _{y \in \overline{V^{\prime}}}\|\partial f(0, y) / \partial y\|$ and get

$$
\|x(n+1)\|=\|f(x(n), y(n))\| \leq \frac{r+1}{2}\|x(n)\|+d\|y(n)\|
$$

where $\overline{V^{\prime}}$ is the closure of the set $V^{\prime}$.

Letting $V=V^{\prime} \cap M$, where $M=\{y \mid\|y\|<(1-r) \rho / 2 d\}$, we have that if $x_{0} \in U, y_{0} \in$ $V, x(n) \in U$, for $n>0$.

According to (4.4), one has

$$
\varlimsup_{n \rightarrow \infty}\|x(n+1)\| \leq \frac{r+1}{2} \varlimsup_{n \rightarrow \infty}\|x(n)\|+d \varlimsup_{n \rightarrow \infty}\|y(n)\| .
$$

By Assumption 1.2, it can be seen that

$$
\varlimsup_{n \rightarrow \infty} y(n)=0
$$

Putting (4.6) into (4.5), one has

$$
\varlimsup_{n \rightarrow \infty}|| x(n+1) \|=0
$$

Hence

$$
\lim _{n \rightarrow \infty}\|x(n+1)\|=0
$$

Therefore the set $U \times V$ is the region of attraction of (1.2).

Now we apply the above theorem to study an example. For simplicity, we strict our attention to the two-dimensional case, and discuss the following system. 
Example 4.2.

$$
x(n+1)=r x(n)+\left(\frac{3}{2}\right) y(n) x(n)^{p}, \quad y(n+1)=\frac{1}{2} y(n),
$$

where $x \in \mathbb{R}, y \in \mathbb{R}, 0<r<1, p>1$.

According to the paper [6], system (4.9) is not globally asymptotically stable. Applying Theorem 4.1 to (4.9), we can easily have the neighborhood $V=\{y|| y \mid<(1-$ $\left.r) / 3 p \rho^{p-1}\right\}$, if $U=\{x|| x \mid<\rho\}$ such that $U \times V$ is the region of attraction of (4.9), and it shows when $p, r$, and $\rho$ become larger, the neighborhood $V$ becomes smaller. Discussing the system with $r=1 / 2, p=2$ studied by [2], we similarly give the neighborhood $V=\{y|| y \mid<1 / 12 \rho\}$ if $U=\{x|| x \mid<\rho\}$ such that $U \times V$ is the region of attraction of the system.

\section{References}

[1] A. Astolfi, New results on the global stabilization of minimum-phase nonlinear systems, Automatica 34 (1998), no. 6, 783-788.

[2] A. Iggidr and M. Bensoubaya, New results on the stability of discrete-time systems and applications to control problems, Journal of Mathematical Analysis and Applications 219 (1998), no. 2, 392414.

[3] D. Nešić and A. Loría, On uniform asymptotic stability of time-varying parameterized discretetime-cascades, IEEE Transactions on Automatic Control 49 (2004), no. 6, 875-887.

[4] R. Sepulchre, Slow peaking and low-gain designs for global stabilization of nonlinear systems, IEEE Transactions on Automatic Control 45 (2000), no. 3, 453-461.

[5] H. J. Sussmann and P. V. Kokotović, The peaking phenomenon and the global stabilization of nonlinear systems, IEEE Transactions on Automatic Control 36 (1991), no. 4, 424-440.

[6] X.-S. Yang, Existence of unbounded solutions of time varying systems and failure of global asymptotic stability in discrete-time cascade systems, IMA Journal of Mathematical Control and Information 22 (2005), no. 1, 80-87.

Xiao-Ming Bai: Department of Mathematics, Huazhong University of Science and Technology, Wuhan 430074, China Current address: Department of Control Science \& Engineering, Huazhong University of Science and Technology, Wuhan 430074, China

Hui-Min Li: Department of Mathematics, Huazhong University of Science and Technology, Wuhan 430074, China

Current address: Department of Control Science \& Engineering, Huazhong University of Science and Technology, Wuhan 430074, China

Xiao-Song Yang: Department of Mathematics, Huazhong University of Science and Technology, Wuhan 430074, China

E-mail address: yangxs@cqupt.edu.cn 


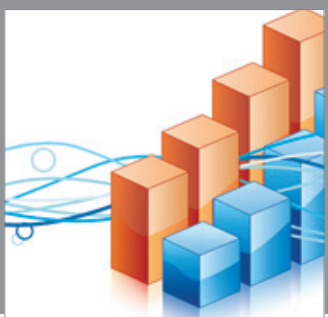

Advances in

Operations Research

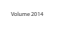

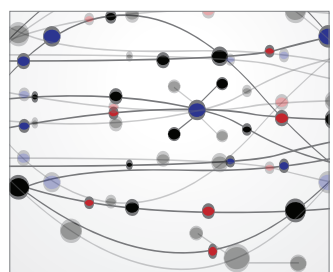

\section{The Scientific} World Journal
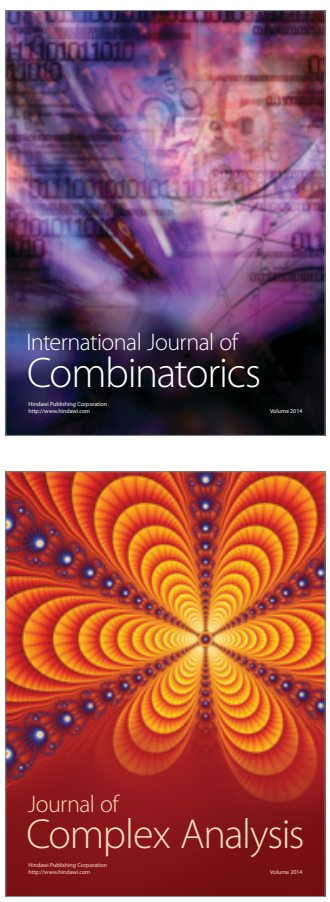

International Journal of

Mathematics and

Mathematical

Sciences
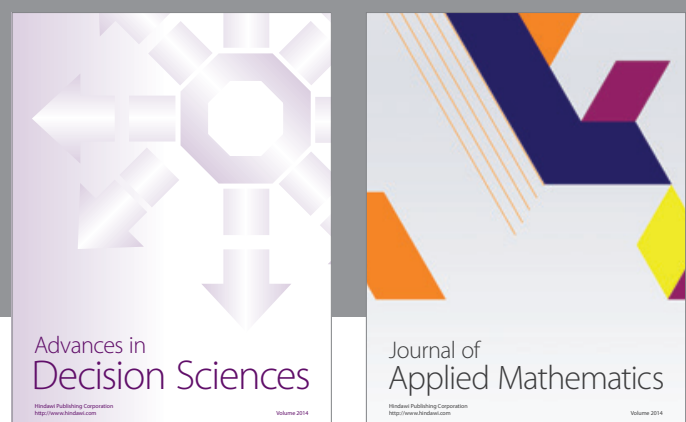

Journal of

Applied Mathematics
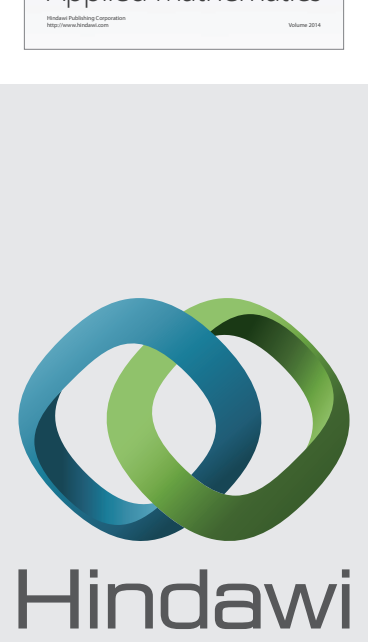

Submit your manuscripts at http://www.hindawi.com
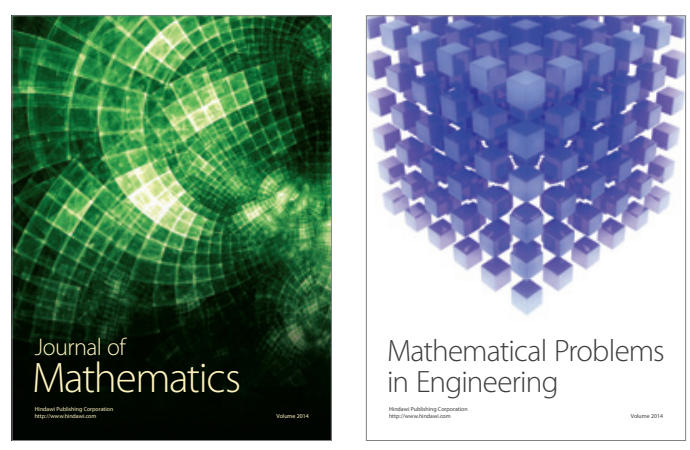

Mathematical Problems in Engineering
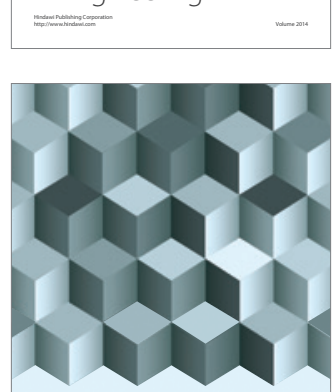

Journal of

Function Spaces
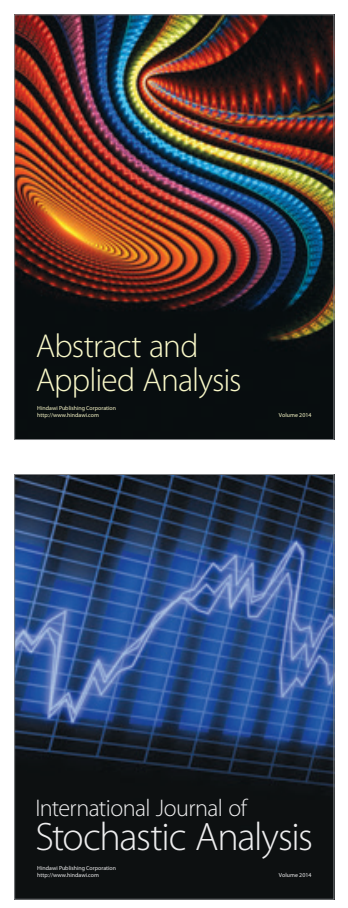

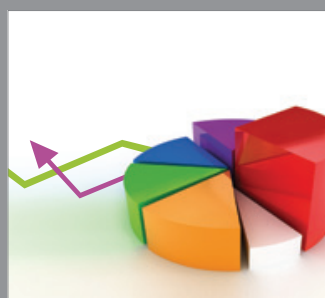

ournal of

Probability and Statistics

Promensencen
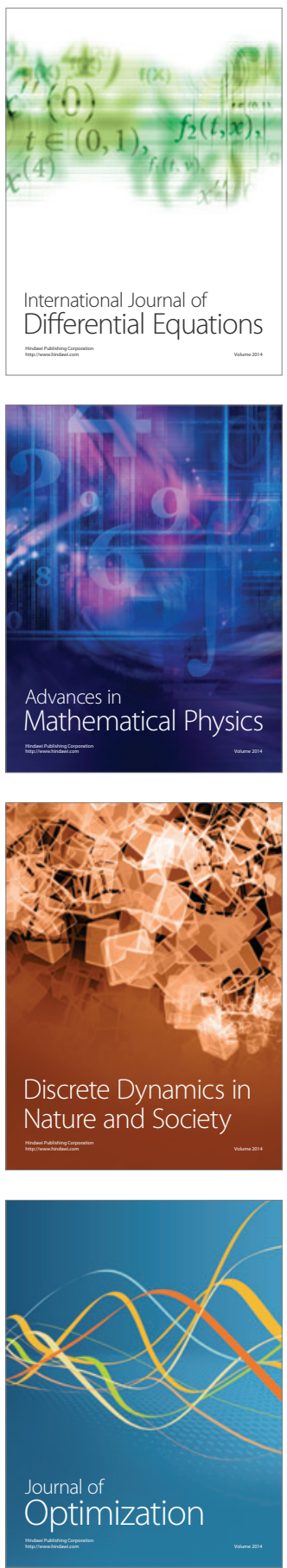